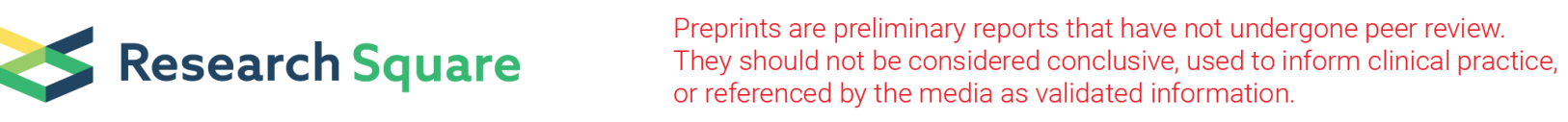

\title{
A score of non-contrast transthoracic echocardiography to detect patent foramen ovale
}

Hui Zhang

Huashan Hospital Fudan University

Haiyan Tang

Huashan Hospital Fudan University

Fei Wu

Huashan Hospital Fudan University

Chun Yu

Huashan Hospital Fudan University

Qiang Dong

Huashan Hospital Fudan University

Wenjie Cao ( $\square$ wenjiecao@fudan.edu.cn )

Huashan Hospital Fudan University https://orcid.org/0000-0003-3438-3882

Research

Keywords: transthoracic echocardiography, patent foramen, stroke, bubble test, transcranial doppler

Posted Date: February 14th, 2020

DOl: https://doi.org/10.21203/rs.2.23613/v1

License: (c) (i) This work is licensed under a Creative Commons Attribution 4.0 International License.

Read Full License 


\section{Abstract}

Background: The aim of this study was to develop a screening score of non-contrast transthoracic echocardiography (TTE) for patent foramen ovale (PFO) in patients with embolic stroke of undetermined source (ESUS). Methods: We performed a retrospective analysis of 218 consecutive patients with a recent ESUS from 2015 to 2018, who received TTE and transcranial Doppler (TCD) as routine examinations. PFO was diagnosed by the bubble test of TCD. Significant differences of the TTE findings between PFO group and non-PFO group were selected into a score. Results: PFO was diagnosed in $35.8 \%(78 / 218)$ of the patients. Compared with non-PFO group, a larger median aortic root diameter (ARd) (34mm vs. $32 \mathrm{~mm}, \mathrm{p}=0.005)$, a lower median peak E wave velocity $(E \mathrm{~m})(61.5 \mathrm{~cm} / \mathrm{s} \mathrm{vs} .68 \mathrm{~cm} / \mathrm{s}, \mathrm{p}=0.005)$ and a lower incidence rate of mitral regurgitation ( $33.8 \%$ vs. $50.7 \%, p=0.016)$ were seen in PFO group. A three-point score of TTE criteria (including aortic root diameter $>33 \mathrm{~mm}, E \mathrm{Em}<72 \mathrm{~cm} / \mathrm{s}$ and without mitral regurgitation, AEM) was tested to be independently predict PFO (odd risk 1.95, 95\% Cl 1.37 2.78, p<0.001). AEM score $\geq 2$ detected PFO with a sensitivity of $0.71(95 \% \mathrm{Cl} 0.67 \rrbracket 0.85)$ and a specificity of 0.53 (95\% $\mathrm{Cl} 0.44$ 0.55). Conclusion: The AEM score measured with non-contrast TTE can select ESUS patients for bubble test of TCD to detect PFO.

\section{Introduction}

Embolic stroke of undetermined source (ESUS), which accounts for approximately 25\% of cases and patent foramen ovale (PFO) is considered to be one of the major causes of ESUS ${ }^{1}$. Ultrasonographic assessment of PFO, using bubble test with transthoracic echocardiograph (TTE), transesophageal echocardiography (TEE) and transcranial Doppler (TCD) remains the diagnostic approach of choice ${ }^{2}$. Although the prevalence of cerebrovascular complications after bubble test were reported very low, stroke risk from paradoxical microbubble embolization can be clinically significant and cannot be guaranteed. ${ }^{3}$ A safe and useful PFO-screening tool before contrast ultrasonographic assessment is important for clinical practice. In this study, we aimed to develop an easily measured screening tool by using noncontrast TTE for PFO in patients with ESUS.

\section{Materials And Methods}

\section{Patients}

We retrospectively reviewed patients diagnosed with acute ESUS from Jan 2015 to Jun 2018. ESUS was defined according to TOAST (Trial of Org 10172 in Acute Stroke Treatment) classification. Demographic characteristics, vascular risk factors (diabetes, hypertension, hyperlipidemia, smoking) and previous stroke history were documented. Ten-point Risk of Paradoxical Embolism (RoPE) score was calculated. ${ }^{4}$

\section{Bubble test}


PFO was diagnosed by the bubble test. ${ }^{5} 9 \mathrm{~mL}$ isotonic saline solution, $1 \mathrm{~mL}$ of air, and 1 drop of the patient's blood were mixed through two $10 \mathrm{~mL}$ syringes connected by a three-way stopcock. The mixture was rapidly injected into the forearm vein during normal respiration and during the maintenance stage of Valsalva maneuver. Right-to-left shunt (RLS) was quantified by counting the number of microbubbles (MBs) within the first 3 cardiac cycles. According to the number of MBs, patients were divided into two groups: PFO group, 1 or more MBs; non-PFO group, no MBs. ${ }^{6}$

\section{TTE}

Parameters of TTE were obtained by retrospectively reviewing the reports. TTE were performed in the left lateral decubitus position using standard imaging planes according to the American Society of Echocardiography recommendations during hospitalization. The aorta root diameters (ARd) and left atria anteroposterior diameters (LAAPd), end-diastolic left ventricle diameters (EDLVd), end-systolic left ventricle diameters (ESLVd), left ventricle ejection fraction (LVEF), peak E-wave velocity (Em) and peak Awave velocity (Am) were collected.

\section{Statistical Analysis}

Statistical analyses were performed using SPSS, version 22 (SPSS Inc, Chicago, IL). P value less than 0.05 was considered to indicate statistical significance. Continuous variables were compared by MannWhitney $\mathrm{U}$ test and categorical variables were compared by Chi-square or Fisher's exact test between PFO and non-PFO groups. Multivariate regression (including variables with $p<0.1$ ) was used to access the association of variables with PFO. Receiver operating characteristic (ROC) analysis was performed to determine the optimal threshold of independent continuous variables in predicting PFO. Base on the previous results, a 3-point score, including 3 independent criteria, was tested by ROC for predicting PFO. This study has been approved by the ethics committee of our institution.

\section{Results}

A total of 218 ESUS patients were finally recruited, median age was 53 years (interquartile range [IQR], $39.75-60), 74.8 \%$ were male (163/218). PFO was detected in $35.8 \%(78 / 218)$ of the patients. No difference was found in baseline characteristics (age, sex, height and weight), risk factors for stroke and RoPE score between PFO group and non-PFO group. Compared with non-PFO group, differences of TTE findings were seen in the PFO group with a larger median ARd (measured at the level of the sinuses of Valsalva) (34 mm vs. $32 \mathrm{~mm}, \mathrm{p}=0.005)$, a lower median Em $(61.5 \mathrm{~cm} / \mathrm{s}$ vs. $68 \mathrm{~cm} / \mathrm{s}, \mathrm{p}=0.005)$ and a lower incidence of mitral regurgitation ( $33.8 \%$ vs. $50.7 \%, p=0016) .($ Table 1$)$ 
Table 1

Univariate analysis between PFO group with Non-PFO group

\begin{tabular}{|c|c|c|c|c|}
\hline Variable & $\begin{array}{l}\text { Total } \\
n=218\end{array}$ & $\begin{array}{l}\text { PFO } \\
n=78\end{array}$ & $\begin{array}{l}\text { Non-PFO } \\
n=140\end{array}$ & $\mathrm{p}$ value \\
\hline Age (yr) (median, IQR) & $53(39.75,60)$ & $52.5(43,59.25)$ & $53(38.25,61)$ & 0.846 \\
\hline Sex (male, \%) & 74.8 & 82.1 & 70.7 & 0.065 \\
\hline Height (cm) & $170(165,173)$ & $170(167,175)$ & $170(164,173)$ & 0.235 \\
\hline Weight (kg) & $68(61.75,75)$ & $67.25(61.875,75)$ & $68(60.25,75)$ & 0.898 \\
\hline Diabetes (\%) & 18.8 & 12.8 & 22.1 & 0.091 \\
\hline Hypertension (\%) & 40.4 & 42.3 & 39.3 & 0.663 \\
\hline Hyperlipidemia (\%) & 17.4 & 17.9 & 17.1 & 0.88 \\
\hline Smoking (\%) & 39.4 & 39.7 & 39.3 & 0.947 \\
\hline Previous stroke (\%) & 17.4 & 16.7 & 17.9 & 0.824 \\
\hline RoPE score (median, IQR) & $5(4,7.25)$ & $6(4,7)$ & $5(4,8)$ & 0.848 \\
\hline \multicolumn{5}{|l|}{ TTE parameter } \\
\hline ARd (mm) (median, IQR) & $33(31,36)$ & $34(31,36.25)$ & $32.5(30,35)$ & 0.005 \\
\hline LAAPd (mm) (median, IQR) & $36(33,38)$ & $36(33,38)$ & $36(33,38)$ & 0.961 \\
\hline EDLVd (mm) (median, IQR) & $49(46,52)$ & $48(45,53)$ & $49(46,52)$ & 0.759 \\
\hline ESLVd (mm) (median, IQR) & $30(28,32)$ & $30(28,32)$ & $30(27,32)$ & 0.961 \\
\hline LEVF (\%) (median, IQR) & $69(65,72)$ & $69(66,72)$ & $69(65.25,72)$ & 0.814 \\
\hline $\mathrm{Em}(\mathrm{cm} / \mathrm{s})$ & $66(53,77.5)$ & $61.5(51,70.25)$ & $68(56,82)$ & 0.005 \\
\hline $\operatorname{Am}(\mathrm{cm} / \mathrm{s})$ & $68(55,80)$ & $67.5(55.5,79)$ & $68.5(55,80)$ & 0.263 \\
\hline Mitral regurgitation (\%) & 45 & 34.6 & 50.7 & 0.022 \\
\hline Tritral regurgitation (\%) & 19.7 & 19.2 & 20 & 0.891 \\
\hline Aortic valve regurgitation (\%) & 20.6 & 23.1 & 19.3 & 0.507 \\
\hline
\end{tabular}

In ROC analysis, ARd predicted PFO with an AUC of $0.61(95 \% \mathrm{Cl} 0.53-0.69, \mathrm{p}=0.005)$ and Em predicted PFO also with an AUC of $0.61(95 \% \mathrm{Cl} 0.53-0.69, \mathrm{p}=0.005)$. Threshold of ARd and Em in predicting PFO 
was $33 \mathrm{~mm}$ and $72 \mathrm{~cm} / \mathrm{s}$ (analyzed with Youden index). Aortic root diameter $>33 \mathrm{~mm}, \mathrm{Em}<72 \mathrm{~cm} / \mathrm{s}$ and no mitral regurgitation were independently associated with PFO, after adjustment of sex and history of diabetes. (Table 2)

Table 2

Univariate and multivariate analysis of the predictors of PFO

\begin{tabular}{|lllll|}
\hline & Univariate & \multicolumn{3}{l|}{ Multivariate* } \\
\hline & $\begin{array}{l}\text { Odds ratio } \\
(95 \% \mathrm{Cl})\end{array}$ & P Value & Odds ratio $(95 \% \mathrm{Cl})$ & P Value \\
\hline $\mathrm{ARd}>33 \mathrm{~mm}$ & $1.99(1.13-3.5)$ & 0.016 & $1.88(1-3.54)$ & 0.049 \\
\hline $\mathrm{Em}<72 \mathrm{~cm} / \mathrm{s}$ & $2.26(1.22-4.18)$ & 0.009 & $2.16(1.13-4.13)$ & 0.02 \\
\hline Without mistral regurgitation & $1.94(1.09-3.44)$ & 0.023 & $1.84(1.01-3.35)$ & 0.046 \\
\hline *Adjusted for sex and history of diabetes & & & \\
\hline
\end{tabular}

A 3-points score system (including ARd $>33 \mathrm{~mm}, \mathrm{Em}<72 \mathrm{~cm} / \mathrm{s}$ and without mitral regurgitation, AEM) predicted PFO with an AUC of $0.65(95 \% \mathrm{Cl} 0.57-0.72, \mathrm{p}<0.001)$. The predictive values of each significance and score were list in Table 3 . AEM $\geq 2$ predicted PFO with a sensitivity of 0.71 and a specificity of 0.53 , which was tested to be the best threshold to predict PFO with Youden index.

Table 3

Sensitivity, Specificity, positive predictive value (PPV) and negative predictive value (NPV) of AEM score for detection of PFO

\begin{tabular}{|c|c|c|c|c|}
\hline & Sensitivity & Specificity & PPV & NPV \\
\hline$A R D>33 \mathrm{~mm}$ & $0.52(0.4-0.63)$ & $\begin{array}{l}0.64(0.55- \\
0.72)\end{array}$ & $\begin{array}{l}0.45(0.34- \\
0.55)\end{array}$ & $0.7(0.62-0.78)$ \\
\hline $\mathrm{Em}<72 \mathrm{~cm} / \mathrm{s}$ & $\begin{array}{l}0.75(0.64- \\
0.84)\end{array}$ & $0.42(0.33-0.5)$ & $0.42(0.33-0.5)$ & $\begin{array}{l}0.75(0.64- \\
0.84)\end{array}$ \\
\hline $\begin{array}{l}\text { Without mitral } \\
\text { regurgitation }\end{array}$ & $\begin{array}{l}0.65(0.53- \\
0.74)\end{array}$ & $0.5(0.42-0.59)$ & $\begin{array}{l}0.42(0.33- \\
0.51)\end{array}$ & $0.72(0.62-0.8)$ \\
\hline With 1 or more factors & $\begin{array}{l}0.94(0.87- \\
0.98)\end{array}$ & $0.15(0.1-0.22)$ & $\begin{array}{l}0.38(0.31- \\
0.45)\end{array}$ & $\begin{array}{l}0.84(0.65- \\
0.95)\end{array}$ \\
\hline With 2 or more factors & $0.71(0.6-0.81)$ & $\begin{array}{l}0.53(0.44- \\
0.62)\end{array}$ & $\begin{array}{l}0.46(0.37- \\
0.55)\end{array}$ & $\begin{array}{l}0.77(0.67- \\
0.85)\end{array}$ \\
\hline With all 3 factors & $\begin{array}{l}0.26(0.17- \\
0.38)\end{array}$ & $\begin{array}{l}0.87(0.81- \\
0.92)\end{array}$ & $\begin{array}{l}0.55(0.38- \\
0.71)\end{array}$ & $\begin{array}{l}0.68(0.61- \\
0.75)\end{array}$ \\
\hline
\end{tabular}

\section{Discussion}


We were able to develop a simple score to predict the detection of PFO in ESUS patients. The AEM score measured with non-contrast TTE could be used to identify high-risk patients for PFO and reduce the use of bubble test. This allows the clinician to easily screen patients at highest risk of PFO for further confirming exams. Moreover, our data addressed the echocardiographic changes of heart structures and functions in PFO patients, which could be useful to explore the potential mechanism of ESUS caused by PFO.

Dilatation of the aortic root may increase the risk of RLS by changing the angulation of the heart in such a way that flow streaming from the inferior vena cava into the right atrium is directed more towards the ostium secundum; thrombotic material is therefore more likely to cross into the systemic circulation, possibly causing a cryptogenic stroke. It has been reported that ARd, marked at the level of the sinuses of Valsalva (34 \pm 4 vs $31 \pm 3 \mathrm{~mm}, \mathrm{p}<0.01$ ), is larger in PFO patients with cryptogenic stroke than in healthy people. ${ }^{7}$ In this study, we compared the ARd in a ESUS patient cohort. All subjects had a homologous profile and our results consisted with previous study, demonstrated a larger median ARd of $34 \mathrm{~mm}$ in PFO patients than those without PFO.

E-wave velocity reflects the left atrial (LA)-left ventricle (LV) pressure gradient during early diastole and is affected by alterations in the rate of LV relaxation and LA. Elevated LA pressure is associated with the absence of RLS in AF stroke patients and may prevent opening of a PFO. ${ }^{8}$ In our study, patients with PFO had lower Em suggest a decreased LA pressure, may associated with a RLS-related stroke.

Mitral regurgitation is the most common valvular heart disorder in high-income countries, and its prevalence increases with age. ${ }^{9}$ In a large-scale cohort of UK adults with 10 years of follow-up, elevated blood presure was continuously associated with an increased risk of mitral regurgitation. ${ }^{10}$ Considering that the association between high blood pressure and mitral regurgitation is similar with that of arteriosclerosis stroke. ESUS patients with no mitral regurgitation may have fewer atherosclerotic risk factors, however, higher likelihood of PFO.

Several limitations of the present study need to be underlined. First, it was a single-center retrospective cohort study with a small sample size. Second, PFO was only diagnosed by the bubble test of TCD, not TEE. Although TEE was considered to be the standard technique for identifying a PFO, some patients were intolerant of this method. Finally, all patients in our study had a stroke. Thus, the differences between those with and without stroke in PFO were not examined, which needs to be addressed in further studies.

\section{Conclusions}

AEM is a risk score based solely on non-contrast TTE parameters that can easily be used to select patients for bubble test to increase the diagnostic yield of PFO after ESUS and might improve the secondary preventive strategy in order to prevent recurrent ischemic strokes. 


\section{Abbreviations}

ESUS: Embolic stroke of undetermined source; PFO: patent foramen ovale; TTE: transthoracic echocardiograph; TEE: transesophageal echocardiography; TCD: transcranial Doppler; RoPE: Risk of Paradoxical Embolism; RLS: Right-to-left shunt; MBs: microbubbles, ARd: aorta root diameters; LAAPd: left atria anteroposterior diameters; EDLVd: end-diastolic left ventricle diameters; ESLVd: end-systolic left ventricle diameters, LVEF: left ventricle ejection fraction ; Em: peak E-wave velocity, Am: peak A-wave velocity; ROC: receiver operating characteristic; LA: left atrial; LV: left ventricle.

\section{Declarations}

\section{Acknowledgements}

None.

\section{Authors' contributions}

WC designed this study. $\mathrm{HZ}$ and $\mathrm{YT}$ draft the manuscript. FW and $\mathrm{CY}$ were involved in image acquisition and analysis in this study. QD carried out the statistical analysis. All authors read and approved the final manuscript.

\section{Funding}

This work was funded by the National Natural Science Foundation of China (Project No. 81901337).

\section{Availability of data and materials}

The data and materials used in this study are available from the corresponding author or the first author on reasonable request.

\section{Ethics approval and consent to participate}

The study was performed with the informed consent of the patient or their next of kin and with ethical approval from the Institutional Review Board of Huashan Hospital.

\section{Consent for publication}

The authors of this study agreed with this manuscript and its submission to Cardiovascular ultrasound.

\section{Competing interests}

The authors declare that they have no competing interests.

\section{References}


1. Hart RG, Diener HC, Coutts SB, et al. Embolic strokes of undetermined source: the case for a new clinical construct. Lancet Neurol 2014;13:429-438.

2. Pearson AC, Labovitz AJ, Tatineni S, Gomez CR. Superiority of transesophageal echocardiography in detecting cardiac source of embolism in patients with cerebral ischemia of uncertain etiology. J Am Coll Cardiol 1991;17:66-72.

3. Romero JR, Frey JL, Schwamm LH, et al. Cerebral ischemic events associated with 'bubble study' for identification of right to left shunts. Stroke 2009;40:2343-2348.

4. Kent $D M$, Ruthazer R, Weimar $C$, et al. An index to identify stroke-related vs incidental patent foramen ovale in cryptogenic stroke. Neurology 2013;81:619-625.

5. Komar M, Olszowska M, Przewlocki T, et al. Transcranial Doppler ultrasonography should it be the first choice for persistent foramen ovale screening? Cardiovasc Ultrasound 2014;12:16.

6. Blersch WK, Draganski BM, Holmer SR, et al. Transcranial duplex sonography in the detection of patent foramen ovale. Radiology 2002;225:693-699.

7. Keenan NG, Brochet E, Juliard JM, et al. Aortic root dilatation in young patients with cryptogenic stroke and patent foramen ovale. Arch Cardiovasc Dis 2012;105:13-17.

8. Aoki J, Kimura K, Iguchi Y, et al. Higher LA pressure may prevent opening of patent foramen ovale in acute ischemic stroke patients with atrial fibrillation. J Neurol Sci 2011;304:111-116.

9. Andell P, Li X, Martinsson A, et al. Epidemiology of valvular heart disease in a Swedish nationwide hospital-based register study. Heart 2017;103:1696-1703.

10. Rahimi K, Mohseni H, Otto CM, et al. Elevated blood pressure and risk of mitral regurgitation: $A$ longitudinal cohort study of 5.5 million United Kingdom adults. PLoS Med 2017;14:e1002404. 